Technical Note

\title{
Influence of Rayleigh effect combined with Marangoni effect on the onset of convection in a liquid layer overlying a porous layer
}

\author{
Rong Liu, Qiu Sheng Liu*, Si Cheng Zhao \\ Institute of Mechanics, Chinese Academy of Sciences, Beijing 100080, China
}

\section{A R T I C L E I N F O}

\section{Article history:}

Received 14 September 2007

Received in revised form 16 June 2008

Available online 19 August 2008

\section{Keywords:}

Porous medium

Instability

Marangoni convection

\begin{abstract}
A B S T R A C T
The coupling mechanism of Rayleigh effect and Marangoni effect in a liquid-porous system is investigated using a linear stability analysis. The eigenvalue problem is solved by means of a Chebyshev tau method. Results indicate that there are three coupling modes between the Rayleigh effect and the Marangoni effect for different depth ratios.
\end{abstract}

(c) 2008 Elsevier Ltd. All rights reserved.

\section{Introduction}

Thermal convection in a horizontal liquid layer is an important problem and has received extensive attention since Bénard [1] observed hexagonal roll cells upon onset of convection in molten spermaceti with a free surface. Rayleigh [2] developed a theory to explain the results of Bénard's experiments. The convection observed in Bénard's experiments is believed to be driven by buoyancy in Rayleigh's theory. In 1956, Block [3] introduced a new viewpoint to explain Bénard's experiments. He demonstrated that the convection cells in a thin liquid layer are driven by variations in surface tension instead of buoyancy. Pearson [4] proposed a new theoretical model different from Rayleigh's and demonstrated analytically that Bénard convection can be driven by variations in surface tension. This surface tension-driven convection is referred as Marangoni-Bénard convection. Nield [5] found that buoyancy and surface tension are tightly coupled in Bénard's experiments. The critical Rayleigh number and the critical Marangoni number are linked by a simple linear relation, i.e.

$R a / R a_{0}+M a / M a_{0}=1$

Here $R a_{0}$ and $M a_{0}$ denote the critical Rayleigh number and the critical Marangoni number for pure buoyancy case and pure thermocapillary case, respectively.

A more complicated problem is the thermal convection driven by buoyancy (Rayleigh effect) and surface tension (Marangoni effect) in a liquid-porous system. This problem has received significant attention since the pioneering works of Horton and Rogers [6],

\footnotetext{
* Corresponding author. Tel.: +861082544095.

E-mail address: liu@imech.ac.cn (Q.S. Liu).
}

and Lapwood [7]. Up to now, most of previous works [8-11] focus on the Rayleigh convection in the liquid-porous system with a rigid upper boundary. In recent years, several investigators have studied the Marangoni convection in the liquid-porous system. Desaive and Lebon [12] firstly used Brinkman's model to study the onset of thermal convection in this system. They reported that the critical depth ratio corresponds to the transition from a liquid layer dominated (short wave) mode to a porous layer dominated (long wave) mode. The critical depth ratio is much smaller in the thermocapillary case (Maranogni convection) than in the buoyancy case (Rayleigh convection).

The instabilities of Marangoni convection and Rayleigh convection have been investigated in many previous works. However, little research has been devoted to the interaction between Rayleigh and Marangoni effects in a fluid-porous system. Thus, for a liquid-porous system subjected to a vertical gradient of temperature, the following question should be asked. If one can obtain an analogy of the Marangoni-Rayleigh instability to Eq. (1) for a liquid-porous case? The relation between the critical Rayleigh number and the Marangoni number for a liquid-porous system is linear, or nonlinear?

Motivated by these previous works and the unknown problem mentioned above, we aim to study the onset of convection driven by the Rayleigh effect combined with the Marangoni effect, especially how these two effects interact under different coupling modes.

\section{The problem formulation and solution procedures}

We consider a homogeneous porous layer of thickness $d_{\mathrm{p}}$ underlying an incompressible liquid layer of thickness $d_{1}$. The combined layers are infinite in the horizontal directions $x, y$. The $z$ direction is opposite to the gravitational acceleration. The interface 
between the fluid and the porous medium is at $z=0$. The bottom of the porous layer is a rigid wall maintained at a constant temperature. The upper boundary is a heat insulating and non-deformable free surface where Marangoni effect can be taken into account. The temperatures of the bottom and upper boundaries are $T_{\mathrm{b}}$ and $T_{\mathrm{u}}$. We use the Brinkman model [13] to describe the porous medium. The governing equations include the continuity, momentum, and energy equations. For the liquid layer, the controlling equations are:

$$
\begin{aligned}
& \nabla \cdot \mathbf{u}_{1}=0 \\
& \frac{\partial \mathbf{u}_{1}}{\partial t}+\mathbf{u}_{1} \cdot \nabla \mathbf{u}_{1}=-\frac{1}{\rho_{1}} \nabla p_{1}-g\left[1-\beta_{1}\left(T_{1}-T_{0}\right)\right] \mathbf{e}_{z}+v_{1} \nabla^{2} \mathbf{u}_{1} \\
& \frac{\partial T_{1}}{\partial t}+\mathbf{u}_{1} \cdot \nabla T_{1}=\kappa_{1} \nabla^{2} T_{1}
\end{aligned}
$$

For the porous layer, the controlling equations are:

$$
\begin{aligned}
\nabla \cdot \mathbf{u}_{\mathrm{p}}=0 & \\
\frac{1}{\phi} \frac{\partial \mathbf{u}_{\mathrm{p}}}{\partial t}= & -\frac{1}{\rho_{\mathrm{l}}} \nabla p_{\mathrm{p}}-g\left[1-\beta_{\mathrm{l}}\left(T_{\mathrm{p}}-T_{10}\right)\right] \mathbf{e}_{z} \\
& \quad+\frac{\mu_{\mathrm{e}}}{\rho_{\mathrm{l}}} \nabla^{2} \mathbf{u}_{\mathrm{p}}-\frac{v_{\mathrm{l}}}{K} \mathbf{u}_{\mathrm{p}} \\
(\rho c)_{\mathrm{p}} \frac{\partial T_{\mathrm{p}}}{\partial t} & +(\rho c)_{1}\left(\mathbf{u}_{\mathrm{p}} \cdot \nabla T_{\mathrm{p}}\right)=\chi_{\mathrm{p}} \nabla^{2} T_{\mathrm{p}}
\end{aligned}
$$

In these equations, the subscripts 1 and $\mathrm{p}$ denote quantities of the liquid layer and the porous layer, respectively. $\mathbf{u}$ denotes the velocity, $p$ the pressure, $T$ the temperature, $t$ the time, $\rho$ the density, $\mu$ the dynamic viscosity, $v$ the kinematic viscosity, $\chi$ the heat conductivity, $\kappa_{\mathrm{l}}$ the thermal diffusivity of the liquid, $\kappa_{\mathrm{p}}$ is defined as $\chi_{\mathrm{p}} /(\rho c)_{\mathrm{l}}, \beta$ the thermal expansion coefficient, $\phi$ the porosity, $c$ the specific heat capacity, $K$ the permeability, $g$ gravitational acceleration. $\mu_{\mathrm{e}}$ is the 'effective viscosity' in the porous medium which is chosen to be equal to the dynamic viscosity of liquid.

To study the stability of thermal convection, the plan is to linearize the equations with respect to a known reference state, i.e., a motionless liquid only with heat conduction. We choose separate length scales for the liquid layer and the porous layer so that both layers are of unit depth in dimensionless form. For the liquid layer, the length is scaled by $d_{1}$, the temperature by $\Delta T_{1} v_{1} / \kappa_{1}$, the time by $d_{1}^{2} / \kappa_{1}$, the velocity by $v_{1} / d_{1}$. For the porous layer, the length is scaled by $d_{\mathrm{p}}$, the temperature by $\Delta T_{\mathrm{p}} v_{\mathrm{l}} / \kappa_{\mathrm{p}}$, the time by $d_{\mathrm{p}}^{2} / \kappa_{\mathrm{p}}$, the velocity by $v_{1} / d_{\mathrm{p}}$. Here $\Delta T_{1}$ and $\Delta T_{\mathrm{p}}$ are the temperature differences in the liquid layer and the porous layer. The disturbances are decomposed into normal modes proportion to $\exp \left[\lambda_{1} t+i k_{1} x\right]$ and $\exp \left[\lambda_{\mathrm{p}}\right.$ $\left.t+i k_{\mathrm{p}} x\right]$ in the liquid and porous layers. Here $\lambda$ is the time growth factor, $k$ is the wave number. Eliminating the pressure fields, the

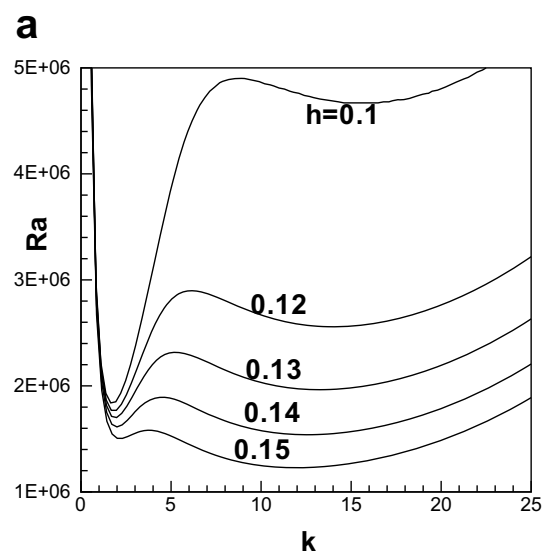

dimensionless linearized normal mode equations for the perturbed variables read as:

$$
\begin{aligned}
& \lambda_{\mathrm{l}} \operatorname{Pr}_{\mathrm{l}}^{-1} \nabla_{\mathrm{l}}^{2} W_{\mathrm{l}}=\nabla_{\mathrm{l}}^{2} \nabla_{\mathrm{l}}^{2} W_{\mathrm{l}}-k_{\mathrm{l}}^{2} R a_{\mathrm{l}} \Theta_{\mathrm{l}} \\
& \lambda_{\mathrm{l}} \Theta_{\mathrm{l}}=W_{\mathrm{l}}+\nabla_{\mathrm{l}}^{2} \Theta_{\mathrm{l}} \\
& \lambda_{\mathrm{p}} \operatorname{Pr}_{\mathrm{p}}^{-1} \phi^{-1} D a \nabla_{\mathrm{p}}^{2} W_{\mathrm{p}}=\Lambda D a \nabla_{\mathrm{p}}^{2} \nabla_{\mathrm{p}}^{2} w_{\mathrm{p}}-\nabla_{\mathrm{p}}^{2} w_{\mathrm{p}}-k_{\mathrm{p}}^{2} R a_{\mathrm{m}} \Theta_{\mathrm{p}} \\
& \lambda_{\mathrm{p}} G \Theta_{\mathrm{p}}=W_{\mathrm{p}}+\nabla_{\mathrm{p}}^{2} \Theta_{\mathrm{p}}
\end{aligned}
$$

At $(z=-1)$, the boundary is a rigid and perfectly heat conduction wall:

$W_{\mathrm{p}}=0, D W_{\mathrm{p}}=0, \Theta_{\mathrm{p}}=0$

At $(z=1)$, the upper surface with surface tension is assumed to be non-deformable and heat insulating:

$W_{\mathrm{l}}=0, D \Theta_{\mathrm{l}}=0, D^{2} W_{\mathrm{l}}+M a_{1} k_{\mathrm{l}}^{2} \Theta_{\mathrm{l}}=0$

At the interface $(z=0)$, continuity of normal and tangential velocities, temperatures, heat flux, and normal momentum and tangential momentum are expressed as:

$$
\begin{aligned}
& h W_{\mathrm{p}}=W_{\mathrm{l}}, h^{2} D W_{\mathrm{p}}=D W_{\mathrm{l}}, \epsilon^{2} \Theta_{\mathrm{p}}=h \Theta_{\mathrm{l}} \\
& \epsilon D \Theta_{\mathrm{p}}=D \Theta_{\mathrm{l}} \\
& \operatorname{Dah}^{4} \Lambda\left(D^{3} W_{\mathrm{p}}-3 k_{\mathrm{p}}^{2} D W_{\mathrm{p}}\right)-h^{4} D W_{\mathrm{p}} \\
& \quad=D a\left(D^{3} W_{\mathrm{l}}-3 k_{\mathrm{l}}^{2} D W_{\mathrm{l}}\right) \\
& \left(D^{2}+k_{\mathrm{l}}^{2}\right) W_{\mathrm{l}}=\Lambda h^{3}\left(D^{2}+k_{\mathrm{p}}^{2}\right) W_{\mathrm{p}}
\end{aligned}
$$

Here $D=\frac{d}{d z}, \nabla_{1}=D^{2}-k_{1}^{2}, \nabla_{\mathrm{p}}=D^{2}-k_{\mathrm{p}}^{2}$. Here all the symbols refer to dimensionless variables. The dimensionless parameters in the above equations include: For the liquid layer, the Rayleigh number defined as $R a_{1}=\beta_{1} g\left(T_{\text {int }}-T_{u}\right) d_{1}^{3} / v_{1} \kappa_{1}$, the Marangoni number defined as $M a_{1}=\sigma_{T}\left(T_{\text {int }}-T_{u}\right) d_{1} / \mu_{1} \kappa_{1}$, the Prandtl number $P r_{1}=v_{1} / \kappa_{1}$. Here $T_{\text {int }}$ is the temperature at the liquid-porous interface. For the porous layer, the Rayleigh number defined as $R a_{\mathrm{p}}=\beta_{\mathrm{l}} \mathrm{g}\left(T_{\mathrm{b}}-T_{\text {int }}\right) d_{\mathrm{p}} K / v_{\mathrm{l}} \kappa_{\mathrm{p}}$, the Prandtl number $\operatorname{Pr}_{\mathrm{p}}=v_{\mathrm{l}} / \kappa_{\mathrm{p}}$. The Darcy number $D a=K / d_{\mathrm{p}}^{2}$, the depth ratio $h=d_{1} / d_{\mathrm{p}}$, the dynamic viscosity ratio $\Lambda=\mu_{\mathrm{e}} / \mu_{\mathrm{l}}$, and the volume heat capacity ratio $G=(\rho c)_{\mathrm{p}} /(\rho c)_{1}, \epsilon=\chi_{\mathrm{l}} / \chi_{\mathrm{p}}$.

These equations and boundary conditions determine an eigenvalue problem which can be solved by Chebyshev tau method $[14,15]$. In our calculation, we choose $\phi=0.3, \epsilon=0.7, D a=$ $9.0 \times 10^{-6}, \operatorname{Pr}=6.0, \Lambda=1.0, G=10.0$.

\section{Results and discussion}

In this section, we will focus on the interaction between the Marangnoni effect and the Rayleigh effect for different coupling

\section{b}

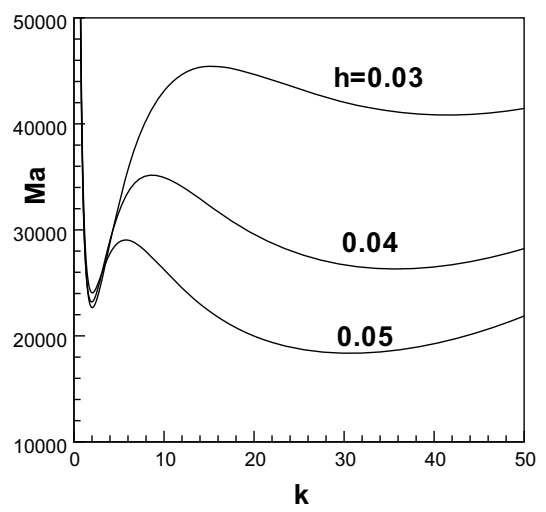

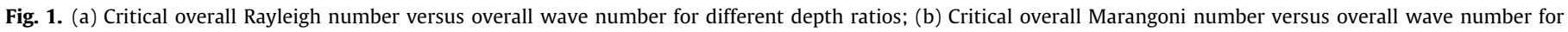
different depth ratios. 
modes. In our problem, the depth ratio $h$ is a major parameter determining the stability modes of the liquid-porous system. In order to compare the results of a porous-liquid system with a pure liquid, we will present our results in the form of the overall Rayleigh number, Marangoni number and wave number for combined layers. The overall Rayleigh number and the overall Marangoni number for the combined layers are defined as:

$R a=\frac{g \beta_{1}\left(T_{b}-T_{u}\right)\left(d_{1}+d_{\mathrm{p}}\right)^{3}}{v_{1} \kappa_{\mathrm{l}}}$,
$M a=\frac{\sigma_{T}\left(T_{b}-T_{u}\right)\left(d_{1}+d_{\mathrm{p}}\right)}{v_{1} \kappa_{\mathrm{l}}}$

The relations between the liquid wave number $k_{1}$, the porous wave number $k_{\mathrm{p}}$ and the overall wave number $k$ are:

$\frac{k_{1}}{d_{1}}=\frac{k_{\mathrm{p}}}{d_{\mathrm{p}}}=\frac{k}{d_{1}+d_{\mathrm{p}}}$

Fig. 1(a) presents the marginal curves of the pure Rayleigh case with a free upper surface. In this figure, for small depth ratio the long wave instability is dominant, and the porous mode convection occurs. With the increase of the depth ratio, the short wave branch becomes more unstable. When short wave mode convection occurs, the circulation is confined in the liquid layer. The critical depth ratio corresponding to such a transition from a long wave mode to a short wave mode is 0.14 . The marginal curves of the pure Marangoni case are shown in Fig. 1(b). For pure Marangoni case, similar transition has also been found. The critical depth ratio for pure Marangoni problem is 0.045 .
We use $h_{\mathrm{R}}$ and $h_{\mathrm{M}}$ to denote the critical depth ratio for pure Rayleigh and pure Marangoni problems, respectively. The Rayleigh effect is dominant in the long wave region when $h<h_{R}$, and in the short wave region when $h>h_{R}$. When $h=h_{R}$, the Rayleigh effect influences the instability in both the long wave and the short wave regions. For the Marangoni effect, similar situation exists. For the porosity $\phi=0.3$, the critical depth ratio $h_{\mathrm{M}}=0.045$ in thermocapillary case and $h_{\mathrm{R}}=0.14$ in gravitation case. Three typical coupling modes of the Rayleigh effect and the Marangoni effect are found in our problem.

For $h<h_{M}$, both the Rayleigh effect and the Marangnoi effect mainly operate in the long wave region, so the coupling instability occurs in the long wave region.

For $h_{M}<h<h_{R}$, the Rayleigh instability occurs in the long wave region, and the Marangoni effect mainly operates in the short wave region. Coupling instability changes from a long wave mode to a short wave mode with the increase of the Marangoni number.

For $h>h_{R}$, both the Rayleigh effect and the Marangnoi effect mainly operate in the short wave region, so the coupling instability occurs in the short wave region.

To understand these three typical coupling modes, we present the marginal curves of the Rayleigh number versus the wave number with different Marangoni numbers for several typical depth ratios $h=0.02,0.10,0.3$.

For $h=0.02$, the instability occurs in the long wave region and corresponding convection is dominated by the combined layers. In the case of $h \ll 1, R a_{1} \sim O\left(d_{1}^{3}\right)$ and $M a_{1} \sim O\left(d_{1}\right)$ are more lower than the critical values. Therefore, the liquid dominated mode is inhibited by the combined layers dominated a

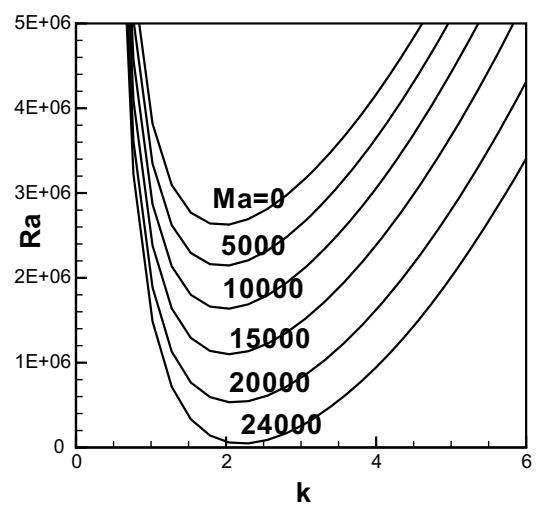

C

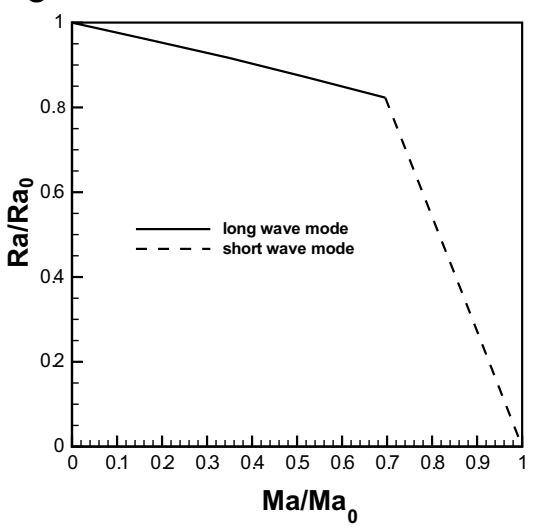

b

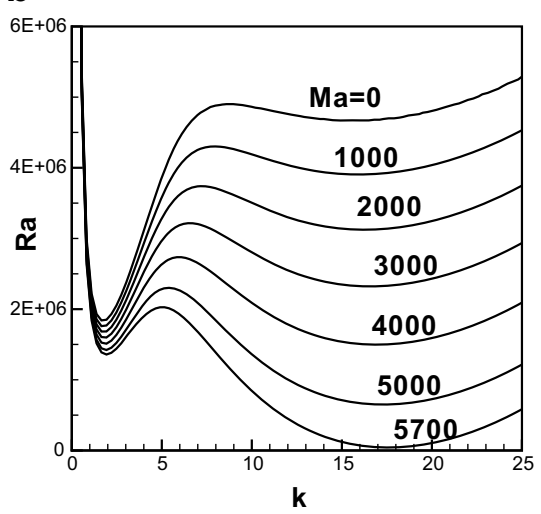

d

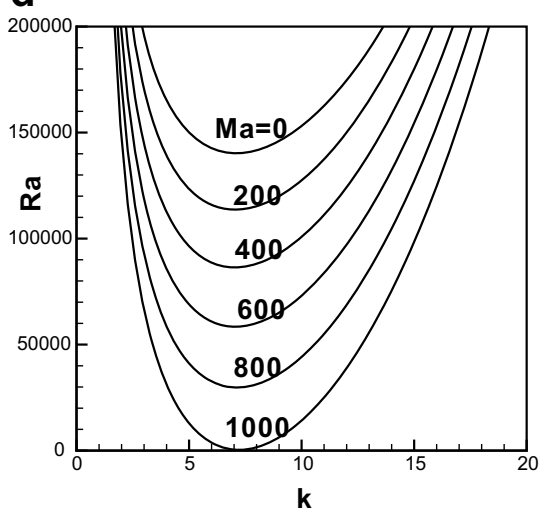

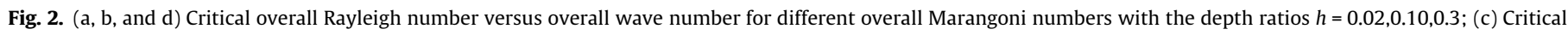
overall Rayleigh number versus overall Marangoni number with the depth ratio $h=0.10$. 
mode. The marginal curves of the Rayleigh number versus wave number are shown in Fig. 2(a). All curves correspond to the combined layers dominated mode. The Rayleigh number decreases with the increase of the Marangoni number. This means the Marangoni effect reinforces the Rayleigh convection dominated by the combined layers. The locus of the critical Rayleigh number and the Marangoni number is a straight line $R a / R a_{0}+M a / M a_{0}=1$, being similar to the case reported by Nield [5] for the Rayleigh-Maranogni convection in a pure liquid. Here $R a_{0}$ is the critical Rayleigh number of pure Rayleigh convection and $M a_{0}$ is the critical Marangoni number for pure Marangoni convection.

For $h=0.10$, the coupling mode is somewhat complicated. The Marangoni effect plays role mainly in the short wave region corresponding the liquid layer dominated mode, whereas the Rayleigh effect plays role mainly in the long wave region corresponding the combined layers dominated mode. The marginal curves of the Rayleigh number versus the wave number are shown in Fig. 2(b). For zero Marangoni number, the long wave branch is the most unstable. In this case, the combined layers dominated mode convection occurs as soon as the Rayleigh number reaches the critical value. We can see that for all wave numbers, the Rayleigh number decreases with the increase of the Marangoni number. The critical Rayleigh number decreases more in the short wave region than in the long wave region. For larger Marangoni numbers, the short wave branch gets more unstable than the long wave branch. In this case, the liquid dominated mode of convection occurs. In Fig. 2(c), the relation between the critical Rayleigh number and the Marangoni number is presented for the case of $h=0.1$. The relation between the critical Rayleigh number and the Marangoni number of a porous-liquid system with $h=0.1$ is segment linear, being different to the classical Rayleigh-Marangoni case of a pure liquid. For the left line segment (solid), the minimum in the long wave branch is smaller than that in the short wave branch, this means the convection is the combined layers dominated mode. For the right line segment (dashed), the minimum in the short wave branch is smaller than that in the long wave branch and the convection is dominated by the liquid layer. $M a / M a_{0}=0.696$ is the critical point switching from the combined layers dominated mode to the liquid layer dominated mode for $h=0.1$. With the increase of the Marangoni number the combined layers dominated mode has the tendency to change into the liquid dominated mode with the convection diminishing in the porous layer. In this transition, the convection in the porous layer is weaken by the presence of Marangoni effect, and diminishes as soon as the liquid layer dominated mode occurs. This result is qualitatively consistent with Saghir's report [16] that the Marangoni convection enhances the flow in the liquid layer and weakens the buoyancy convection in the porous layer.

As shown in Fig. 2(d) for $h=0.3$, the long wave branch disappears for all Marangoni numbers. The convection is dominated by the liquid layer when instability occurs. The porous layer only influences the heat boundary condition at the liquid-porous interface. In this situation, the convection reduces to the classical Rayleigh-Marangoni case. The relation between the critical Rayleigh number and the Marangoni number is linear.

\section{Summary}

A linear instability analysis is proposed to study the thermal convection in a liquid-porous system. We find three typical coupling modes when the Rayleigh effect is combined with the Marangoni effect. For $h<h_{M}$, the combined layers dominated instability (long wave mode) occurs. The Marangoni effect enhances the flow in both layers. For $h_{M}<h<h_{R}$, the combined layers dominated (long wave) mode changes into the liquid dominated (short wave) mode with the increase of the Marangoni number. The Marangoni effect enhances the convection in the liquid layer and weakens the flow in the porous layer. For $h>h_{R}$, only the liquid dominated convection occurs. The Marangoni effect has no influence on the flow in the porous layer.

\section{Acknowledgements}

The authors thank the financial support for this research from National foundation of China(10772185,10372105) and the Knowledge Innovation Program of Chinese Academy of Sciences (KGCX-SW-409).

\section{References}

[1] H. Bénard, Rev. Gen. Sci. Pure Appl. 11 (1900) 1261.

[2] L. Rayleigh, Philos. Mag. 32 (6) (1916) 529.

[3] M.J. Block, Nature 178 (1956) 650.

[4] J.R.A. Pearson, J. Fluid Mech. 4 (1958) 489.

[5] D.A. Nield, J. Fluid Mech. 19 (1964) 341.

[6] C.W. Horton, G.T. Rogers, J. Appl. Phys. 16 (1945) 367

[7] E.R. Lapwood, Proc. Camb. Philos. Soc. 44 (1948) 508.

[8] D.A. Nield, J. Fluid Mech. 8 (1977) 513.

[9] W.J. Sun, Convective Instability in Superposed Porous an Free Layer, Ph.D. Dissertation, University of Minnesota, Minneapolis, 1973.

[10] F. Chen, C.F. Chen, J. Heat Transf. 110 (1988) 403.

[11] F. Chen, C.F. Chen, J. Fluid Mech. 234 (1992) 97.

[12] T. Desaive, G. Lebon, Phys. Rev. E 64 (2001) 066304-1.

[13] H.C. Brinkman, Appl. Sci. Res. A1 (1947) 27.

[14] B. Straughan, J. Comput. Phys. 173 (2001) 320.

[15] S.A. Orszag, J. Fluid Mech. 50 (1971) 689.

[16] M.Z. Saghir, P. Comi, M. Mehrvar, Int. J. Therm. Sci. 41 (2002) 207. 